\title{
Optical measurement of cerebral hemodynamics and oxygen metabolism in neonates with congenital heart defects
}

\author{
Turgut Durduran \\ ICFO-Institut de Ciències Fotòniques \\ Av Canal Olimpic s/n \\ Castelldefels, 08860 Spain \\ Chao Zhou \\ Erin M. Buckley \\ Meeri N. Kim \\ University of Pennsylvania \\ Department of Physics and Astronomy \\ Philadelphia, Pennsylvania 19104
}

\section{Guoqiang Yu}

University of Kentucky

Center for Biomedical Engineering

Lexington, Kentucky 40506

\section{Regine Choe}

University of Pennsylvania

Department of Physics and Astronomy

Philadelphia, Pennsylvania 19104

\section{J. William Gaynor}

Thomas L. Spray

Children's Hospital of Philadelphia

Division of Cardiothoracic Surgery

Philadelphia, Pennsylvania 19104

\section{Suzanne M. Durning}

Children's Hospital of Philadelphia

Division of Respiratory Therapy

Philadelphia, Pennsylvania 19104

\section{Stefanie E. Mason}

Children's Hospital of Philadelphia

Division of Neurology

Philadelphia, Pennsylvania 19104

\section{Lisa M. Montenegro}

\section{Susan C. Nicolson}

Children's Hospital of Philadelphia

Department of Anesthesiology and Critical Care

Philadelphia, Pennsylvania 19104

\section{Robert A. Zimmerman}

Children's Hospital of Philadelphia

Division of Neuroradiology

Philadelphia, Pennsylvania 19104

\section{Mary E. Putt}

University of Pennsylvania

Department of Biostatistics and Epidemiology

Philadelphia, Pennsylvania 19104

\section{Jiongjiong Wang}

University of Pennsylvania

Department of Radiology

Philadelphia, Pennsylvania 19104
Joel H. Greenberg

University of Pennsylvania

Department of Neurology

Philadelphia, Pennsylvania 19104

John A. Detre

University of Pennsylvania

Department of Radiology and

Department of Neurology

Philadelphia, Pennsylvania 19104

\section{Arjun G. Yodh}

University of Pennsylvania

Department of Physics and Astronomy

Philadelphia, Pennsylvania 19104

\section{Daniel J. Licht}

Children's Hospital of Philadelphia

Division of Neurology

Philadelphia, Pennsylvania 19104 and

University of Pennsylvania

Department of Neurology

Philadelphia, Pennsylvania 19104

\begin{abstract}
We employ a hybrid diffuse correlation spectroscopy (DCS) and near-infrared spectroscopy (NIRS) monitor for neonates with congenital heart disease $(n=33)$. The NIRS-DCS device measured changes during hypercapnia of oxyhemoglobin, deoxyhemoglobin, and total hemoglobin concentrations; cerebral blood flow $\left(\mathrm{rCBF}_{D C S}\right)$; and oxygen metabolism $\left(\mathrm{rCMRO}_{2}\right)$. Concurrent measurements with arterial spin-labeled magnetic resonance imaging $\left(\mathrm{rCBF}_{A S L-M R l}, n=12\right)$ cross-validate $\mathrm{rCBF}_{D C S}$ against $\mathrm{rCBF}_{A S L-M R l}$, showing good agreement $(R=0.7, p=0.01)$. The study demonstrates use of NIRSDCS on a critically ill neonatal population, and the results indicate that the optical technology is a promising clinical method for monitoring this population. ( 2010 Society of PhotoOptical Instrumentation Engineers. [DOI: 10.1117/1.3425884]
\end{abstract}

Keywords: near-infrared spectroscopy; diffuse optics; diffuse correlation spectroscopy; congenital heart disease; pediatrics; cerebral blood flow.

Paper 09377RR received Aug. 26, 2009; revised manuscript received Mar. 2, 2010; accepted for publication Mar. 12, 2010; published online May 24, 2010.

\section{Introduction}

Approximately 30,000 infants are born each year in the United States with congenital heart disease (CHD), with about a third requiring major surgical repair in the first few months of life. ${ }^{1}$ Recent advances in cardiac surgery for complex CHD have minimized infant mortality. Thus, the current focus in

$1083-3668 / 2010 / 15(3) / 037004 / 10 / \$ 25.00$ C 2010 SPIE 
the clinical community is oriented toward preventing neurologic injury and improving neurocognitive outcome in these high-risk babies who grow up to face various medical and academic challenges. ${ }^{2,3}$

Two key determinants of neurologic injury in babies with complex CHD are now believed to be damaged cerebral autoregulation $^{4}$ and low-baseline cerebral blood flow $\left(\mathrm{CBF}_{b l}\right) .{ }^{5}$ Cerebral autoregulation ${ }^{6}$ is loosely defined as the ability of a subject to maintain stable and adequate blood flow to the brain at the microvascular level despite changes in cerebral perfusion pressure. Damaged cerebral autoregulation may complicate the clinical management of sick newborns, where the goal is to minimize periods of hypoxia and hypoperfusion. In fact, damaged cerebral autoregulation implies that the local CBF may be passively dependent on external factors. Therefore, to achieve success with this approach to clinical management, it is desirable to continuously monitor cerebral autoregulation and local $\mathrm{CBF}$ at the bedside and optimize patient management accordingly.

Unfortunately, it is very difficult to measure cerebral autoregulation noninvasively at the bedside. ${ }^{7}$ The neonatal population presents challenges different from those of adults. Traditional modalities for measurement of CBF in adults (PET, SPECT, Xenon CT, ASL-MRI, Doppler ultrasound) often pose safety risks, require patient transport, or are limited to large-vessel measurements. ${ }^{8-10}$ Thus, an unfilled niche exists for a safe, noninvasive, continuous, bedside monitor of CBF and related hemodynamics in the infant microvasculature.

In this study, we focus on infants born with CHD. We validate a new diffuse optical diagnostic technique, diffuse correlation spectroscopy (DCS), against a more established modality, arterial spin-labeled magnetic resonance imaging (ASL-MRI), for measurement of cerebral blood flow (CBF). Then, using an all-optical instrument that combines DCS with near-infrared spectroscopy (NIRS), we derive changes in oxy- and deoxyhemoglobin concentrations, CBF, as well as a calculated estimate of cerebral metabolic rate of oxygen extraction $\left(\mathrm{CMRO}_{2}\right)$ during hypercapnia.

Previously, optical monitoring using near-infrared light (NIRS) has been used for transcranial measurements of total hemoglobin concentration, blood oxygen saturation. ${ }^{11,12}$ NIRS is particularly successful in infants due to their thin skulls. ${ }^{13-20}$ NIRS has also been used for CBF monitoring using exogenous tracers such as indocyanine green or changes in inspired gases. ${ }^{21,22}$ Unfortunately, this approach using tracers is indirect at best and can be limited in certain physiological conditions. ${ }^{23}$

A recent advance in biomedical optics has been the development and in vivo application of diffuse correlation spectroscopy (DCS) ${ }^{24-27}$ DCS measures microvascular blood flow in deep tissue utilizing the temporal intensity fluctuations of multiply scattered light. DCS is based on physical principles somewhat similar to those of NIRS and thus shares advantages such as noninvasiveness and the ability to penetrate to deep tissues. Additionally, DCS provides a direct measure of $\mathrm{CBF}$ without the need for exogenous tracers. ${ }^{28-30}$ DCS has been validated against other modalities under a variety of conditions, ${ }^{31-36}$ and concurrent use of DCS and NIRS in hybrid probes offers potential for continuous noninvasive estimation of $\mathrm{CMRO}_{2}$. $^{28,29,35,37-39}$
However, application of DCS in neonatal populations has been very limited. For example, while NIRS-DCS has been utilized in adults ${ }^{40}$ and premature infants ${ }^{33,35}$ in clinical settings, it has not as yet been validated or even explored as a monitor of cerebral hemodynamics in critically ill neonates with low-baseline $\mathrm{CBF}^{5}$

For this study, we measure CBF in infants with complex forms of CHD. During the study, we employ increased carbon dioxide $\left(\mathrm{CO}_{2}\right)$ in the inspired gas mixture as an intervention to study vascular reactivity in the population. Information about vascular reactivity, in turn, permits us to assess the status of cerebral autoregulation. ${ }^{5}$ Increased $\mathrm{CO}_{2}$ is a potent cerebral arteriolar vasodilator. Healthy response to hypercapnia is characterized by a slight increase in blood pressure, by a drop in vascular resistance, and by an increase in $\mathrm{CBF}^{41}$ The $\mathrm{CBF}$ response to $\mathrm{CO}_{2}$ is a marker for physiologic reserve in the cerebrovascular bed. $\mathrm{CO}_{2}$ reactivity [i.e., change in $\mathrm{CBF}$ per change in partial pressure of $\left.\mathrm{CO}_{2}\left(\mathrm{pCO}_{2}\right)\right]$ is of interest, because impaired $\mathrm{CO}_{2}$ reactivity has been associated with poor neurodevelopmental outcome and a higher risk of death in all age groups. ${ }^{4-44}$

Neonates with complex forms of CHD are dependent on a patent ductus arteriosus for systemic blood flow, including $\mathrm{CBF}$. In these neonates, management of the delicate balance of pulmonary blood flow and systemic blood flow is critical. Since $\mathrm{CO}_{2}$ is also a potent pulmonary arteriolar vasoconstrictor, its presence can alter this balance by limiting pulmonary flow in favor of systemic circulation. Increased inspired $\mathrm{CO}_{2}$ $\left(\mathrm{FiCO}_{2}\right)$ has been shown by NIRS to significantly increase mixed venous oxygenation in neonates with hypoplastic left heart syndrome (HLHS) ${ }^{45}$ and to increase CBF during hypothermic cardiopulmonary bypass. ${ }^{46,47}$ Studies from our institution have demonstrated that periventricular leukomalacia (PVL), a form of white matter injury seen in this patient population and in infants born prematurely, occurred in $28 \%$ of CHD neonates and was associated with lower baseline CBF values and a smaller change in cerebral blood flow with hypercapnia-i.e., associated with reduced $\mathrm{CO}_{2}$ reactivity. ${ }^{5}$ We note that while the baseline $\mathrm{CBF}$ of term neonates born with $\mathrm{CHD}$ as a group $(10.2 \pm 4.4 \mathrm{ml} / 100 \mathrm{~g} / \mathrm{min})$ also tends to be lower than healthy neonates ${ }^{48}$ $(16.6 \pm 5.9 \mathrm{ml} / 100 \mathrm{~g} / \mathrm{min})$, the key point we stress here is that those with lower CBF among CHD neonates had a higher occurrence of neurological injury. ${ }^{5}$

Very recently, we have demonstrated in a subpopulation of infants with CHD that neurological injury was associated with decreased blood oxygen saturation and increased time to surgery, thus indicating the potential value for preoperative monitoring. ${ }^{49}$ NIRS has also been used to follow hemodynamic changes in CHD neonates after the Norwood procedure, suggesting that cerebral hemodynamics were influenced by external interventions and postoperative events. ${ }^{50}$ The present feasibility study demonstrates potential for relating neurological outcome and cerebral hemodynamics in this early period after surgery by demonstrating the use of an alloptical, bedside monitor during this presurgical period that could safely be deployed at the bedside. We measure hemodynamic $\mathrm{CO}_{2}$ reactivity in response to induced hypercapnia, which, as mentioned earlier, could be related to the neurological outcome (to be demonstrated in a future, larger study). 
Table 1 Tabulation of various characteristics of the subjects. Values are quoted as mean+standard error of the mean. Admission CHD diagnosis of either hypoplastic left heart syndrome (HLHS) or +transposition of the great arteries (TGA) was required for study inclusion.

\begin{tabular}{cccccc}
\hline Diagnosis & Gender & $\begin{array}{c}\text { Gestational Age } \\
(\text { week })\end{array}$ & $\begin{array}{c}\text { Age at Study } \\
(\text { day })\end{array}$ & $\begin{array}{c}\text { Head Circumference } \\
(\mathrm{cm})\end{array}$ & $\begin{array}{c}\text { Birth Weight } \\
(\mathrm{kg})\end{array}$ \\
\hline TGA & $6 \mathrm{M} / 5 \mathrm{~F}$ & $39.4 \pm 0.2$ & $4.5 \pm 0.5$ & $34.8 \pm 0.2$ & $3.6 \pm 0.1$ \\
HLHS & $13 \mathrm{M} / 9 \mathrm{~F}$ & $38.8 \pm 0.2$ & $3.7 \pm 0.3$ & $34.3 \pm 0.2$ & $3.3 \pm 0.1$ \\
\hline
\end{tabular}

Furthermore, arguably, addition of a CBF measure and calculation of $\mathrm{CMRO}_{2}$ should further enhance this potential by providing a more complete picture of the cerebral oxygen metabolism than currently available.

The present work is the first to report the use of such an all-optical instrument in neonates with complex CHD $(n=33)$. Furthermore, concurrent measurements with ASLMRI $\left(\mathrm{rCBF}_{A S L-M R I}\right)$ in $12(n=12)$ neonates cross-validate DCS $\left(\mathrm{rCBF}_{D C S}\right)$ against $\mathrm{rCBF}_{A S L-M R I}$. The optical data is compared to literature values of vasoreactivity to hypercapnia, and a calculated index that is approximately proportional to changes in $\mathrm{CMRO}_{2}$ during hypercapnia is determined.

\section{Materials and Methods}

\subsection{Population}

With institutional review board approval, all newborn infants with complex CHD admitted to the cardiac intensive care unit (CICU) at Children's Hospital of Philadelphia (CHOP) were screened for study inclusion and were approached for participation if the admitting CHD diagnosis was hypoplastic left heart syndrome (HLHS) or transposition of the great arteries (TGA). All patients $(n=33)$ were at full-term age ( $40 \pm 4$ weeks gestation age) with pre- or postnatally diagnosed CHD and were scheduled for surgery with with cardiopulmonary bypass with or without deep hypothermic circulatory arrest. A full baseline neurologic examination was carried out by a child neurologist (DJL) on the day prior to the surgery. Table 1 shows detailed tabulation of the patient characteristics.

\subsection{Study Protocol}

All procedures were approved by the Children's Hospital of Philadelphia Institutional Review Board. On the morning of surgery, all patients were transported to the operating room for induction of general anesthesia (fentanyl 5-10 $\mu \mathrm{g} / \mathrm{kg}$, pancuronium $0.2 \mathrm{mg} / \mathrm{kg}$ ). Vital signs, including blood pressure, electrocardiogram, transcutaneous oxygen saturations, and end-tidal $\mathrm{CO}_{2}\left(E t \mathrm{CO}_{2}\right)$ measurements, were monitored during the induction of anesthesia, in transport, and while in the MRI. On arrival at the MRI suite, arterial and venous blood samples were drawn for baseline arterial $\mathrm{CO}_{2}\left(\mathrm{PaCO}_{2}\right)$ and co-oximetry (quantitative venous and arterial oxygen saturations). The protocol has been previously described, ${ }^{5,49,51}$ and a time line is outlined in Fig. 1.

\subsection{Diffuse Optics: Background, Instrumentation, and Analysis}

In the near-infrared spectral region, light is multiply scattered as it travels centimeters through deep tissue. Photon absorption in the near-infrared range also occurs mainly due to oxyand deoxyhemoglobin, water, and lipid. A detectable amount of light scattering comes from red blood cells (RBCs). If photons are scattered from moving $\mathrm{RBCs}$, then the light intensity interference pattern (i.e., the speckle pattern) on the tissue surface will fluctuate in time. The resultant fluctuations of the detected intensity are measured by DCS. NIRS, on the other hand, measures the differential change in the transmitted light intensity at multiple wavelengths due to absorption and scattering, which, in turn, depend on concentrations of oxy- and deoxyhemoglobin $\left(\Delta \mathrm{HbO}_{2}\right.$ and $\Delta \mathrm{Hb}$, respectively) among other factors.

The present investigation employs a hybrid instrument combining NIRS and DCS. ${ }^{28,38}$ For DCS, we employed a long-coherence-length laser at $785 \mathrm{~nm}$. Three lasers $(690 \mathrm{~nm}$, $785 \mathrm{~nm}, 830 \mathrm{~nm}$ ) modulated at $70 \mathrm{MHz}$ were used for NIRS. For DCS, two high-sensitivity avalanche photodiode detectors and a correlator board were used to calculate intensity autocorrelation functions in real time. For NIRS, a homodyne detection scheme with one detector channel was used.

As shown in Fig. 2, a probe with one source fiber (shared by NIRS and DCS lasers), two detector fibers for DCS, and one detector fiber for NIRS was used. All detectors were placed at $2.5 \mathrm{~cm}$ away from the source fiber. The probe thickness was $\sim 2.5 \mathrm{~mm}$, and fibers were $12 \mathrm{~m}$ long. All materials

\section{Protocol Time Line (45-60 Minutes)}

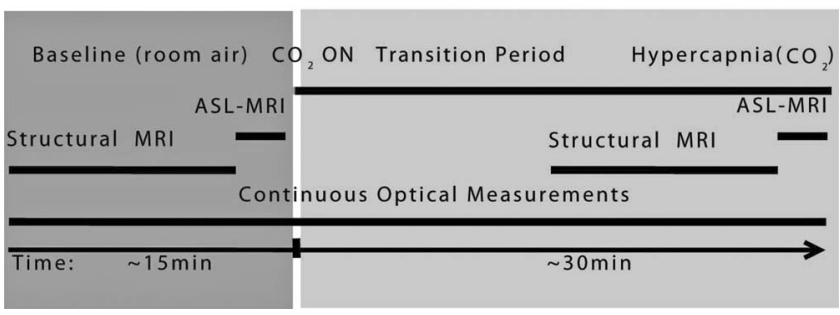

Fig. 1 Measurement time line consists of two periods: (1) Baseline, where the subject is breathing room air equivalent mixture with zero $\mathrm{CO}_{2}$ concentration, and (2) Hypercapnia, where $\mathrm{CO}_{2}$ is added to the mixture. There is an intermediate transition period until significant changes are recorded in the $\mathrm{EtCO}_{2}$ measurements. Optical data is obtained continuously throughout the study. Structural MRIs are obtained in both periods prior to ASL-MRI measurements. 
Concurrent ASL-MRI \&

Optical Measurement

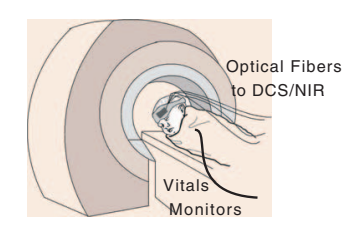

Optical Probe:

Face View

0
2.5
0

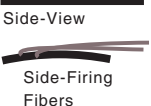

Fibers

Source: NIRS + DCS

(shared)

Detector: NIRS

Detector: DCS (X2)

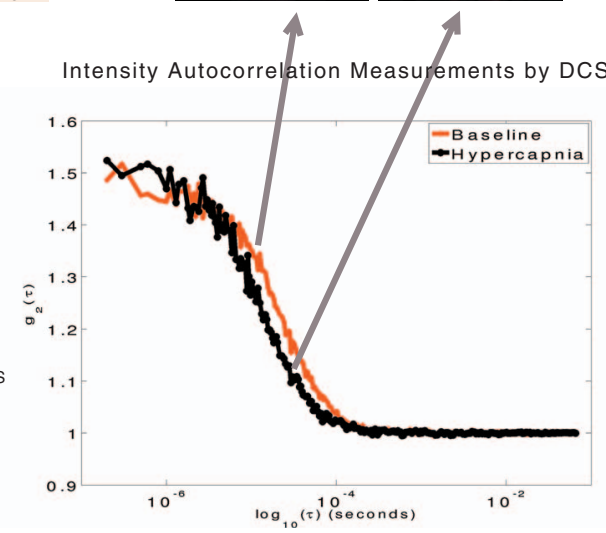

Fig. 2 Measurement schematic showing optical probe details, probe placement, and the concurrent measurement. Typical ASL-MRI images (brighter red/orange indicating higher blood flow) and autocorrelation functions measured by DCS during baseline and hypercapnia are also shown.

were thoroughly tested for MRI compatibility. Fiducial markers were placed over the probe to locate fiber positions in the MRI images.

Optical data was analyzed using a semi-infinite, homogeneous medium model that is expected to be fairly accurate given the thin neonate skulls. We have verified this assumption with simulations based on a two-layer model. ${ }^{28}$ Any variation in the tissue optical properties or tissue dynamics within the probed volume, such as those resulting from a CBF change due to $\mathrm{CO}_{2}$ inhalation, are detectable in the decay rate of the intensity autocorrelation function $\left[g_{2}(\tau)\right]$ and in the amplitude and phase of the NIRS signal. Figure 2 shows two representative $g_{2}(\tau)$ measurements wherein changes in decay time due to increased $\mathrm{CBF}$ during hypercapnia are clearly visible. The semi-infinite model was iteratively fit to the measured autocorrelation functions, and a flow-index from the decay time for each hemisphere was extracted every $8 \mathrm{~s}$. For NIRS, a modified Beer-Lambert equation with assumed photon path lengths from the literature was used. ${ }^{52-54}$ NIRS calculations report $\mathrm{HbO}_{2}$ and $\mathrm{Hb}$ concentration changes relative to the baseline (i.e., $\Delta \mathrm{HbO}_{2}, \Delta \mathrm{Hb}, \Delta \mathrm{THC}$ ). Note that measured changes in absorption from NIRS data were used to improve the DCS fits. DCS flow indices from two detectors were divided by the baseline values and averaged, providing a measure of $\mathrm{rCBF}_{D C S}$ versus time. All analysis procedures have been previously described. ${ }^{40}$

Changes in $\mathrm{CMRO}_{2}\left(r C M R \mathrm{O}_{2}\right)$ can be calculated from a synthesis of $r C B F, \triangle H b$, and $\triangle T H C$ utilizing a relatively simple model. ${ }^{37,55-57}$ In particular, a compartmentalized model of the vasculature is assumed, and an equation that relates these measurable quantities is derived using Fick's law: $C M R \mathrm{O}_{2}=\mathrm{O} E F \times C B F \times C_{a}($ Ref. 41$) . \mathrm{O} E F$ is the normalized oxygen extraction fraction-i.e., the difference between oxygen concentrations in arterial $\left(C_{a}\right)$ and venous ends of the vasculature. Since diffuse optical signal mainly originates from the microvasculature, further assumptions are made to relate the microvascular blood oxygenation to the percentage of blood in the venous and arterial components. These assumptions lead to an equation that has been used ${ }^{28,29,37,38}$ to estimate $\mathrm{rCMRO}_{2}$ :

$$
\begin{gathered}
r C M R \mathrm{O}_{2}=r \mathrm{O} E F \times r C B F, \\
=\left(\frac{S_{a} \mathrm{O}_{2}-S_{t} \mathrm{O}_{2}}{S_{a} \mathrm{O}_{2 b l}-S_{t} \mathrm{O}_{2 b l}}\right)\left(\frac{\gamma_{b l} S_{a} \mathrm{O}_{2 b l}}{\gamma S_{a} \mathrm{O}_{2}}\right) r C B F, \\
\approx\left(\frac{S_{a} \mathrm{O}_{2}-S_{t} \mathrm{O}_{2}}{S_{a} \mathrm{O}_{2 b l}-S_{t} \mathrm{O}_{2 b l}}\right)\left(\frac{S_{a} \mathrm{O}_{2 b l}}{S_{a} \mathrm{O}_{2}}\right) r C B F .
\end{gathered}
$$

$\mathrm{S}_{t} \mathrm{O}_{2}=\mathrm{HbO}_{2} /(T H C)$ is the microvascular blood oxygen saturation measured by NIRS. Subscript $b l$ is used throughout this paper to indicate baseline values of a parameter. Baseline $S t \mathrm{O}_{2}\left(S_{t} \mathrm{O}_{2 b l}\right)$ was assumed from literature values for neonates to be $65 \% .^{58-62} T H C_{b l}$ is also assumed from baseline values reported in the literature as $53 \mu \mathrm{M} .^{58-62}$ Changes in $\mathrm{HbO}_{2}$ and $\mathrm{Hb}$ concentrations were then used to calculate the hypercapnic values for $S t \mathrm{O}_{2}$ and $T H C$. Note that we do not estimate THC from systemic measures of hemoglobin concentration, since it has been previously shown that NIRS values in CHD populations may not be directly correlated to systemic measures of hemoglobin. ${ }^{62}$

Here, $\gamma$ is the percentage of blood in the venous compartment. In the last step, we assume that $\gamma$ does not change with hypercapnia in accordance with previous observations of neonates and children with CHD that hypercapnia does not alter the proportion of arterial to venous blood in the brain. ${ }^{63}$

Last, in order to report a single-relative change per parameter per subject, we have used the $\mathrm{EtCO}_{2}$ data to define a stable baseline and a stable hypercapnic period. The latter was defined as the time period during increased $\mathrm{CO}_{2}$ administration where $\mathrm{EtCO}_{2}$ was at a plateau. All the reported changes in both optical and systemic data are calculated according to these time periods. All \% changes are reported as \% of baseline.

\subsection{MRI Imaging Protocol}

Due to various technical problems, either with ASL-MRI or optical data acquisition, high-quality data were acquired concurrently with custom pediatric ASL-MRI sequences in 12 of the 33 neonates. ${ }^{5,64-66}$ Figures 1 and 2 show the time line and representative pre- and during hypercapnia ASL-MRI images. All MRIs were acquired on a Siemens 3.0T Trio at Children's Hospital of Philadelphia. In particular, MRI sequences included multiplanar reconstructed (MPR) volumetric $\mathrm{T}_{1}$ and $\mathrm{T}_{2}$ SPACE (short for sampling perfection with applicationoptimized contrasts using different flip-angle evolutions) sequences acquired in the axial plane and later reconstructed in the sagittal and coronal planes. Axial fluid attenuated inversion recovery (FLAIR), susceptibility (both standard echo gradient and susceptibility weighted imaging), and diffusion weighted imaging (DWI) sequences were also acquired. Clini- 

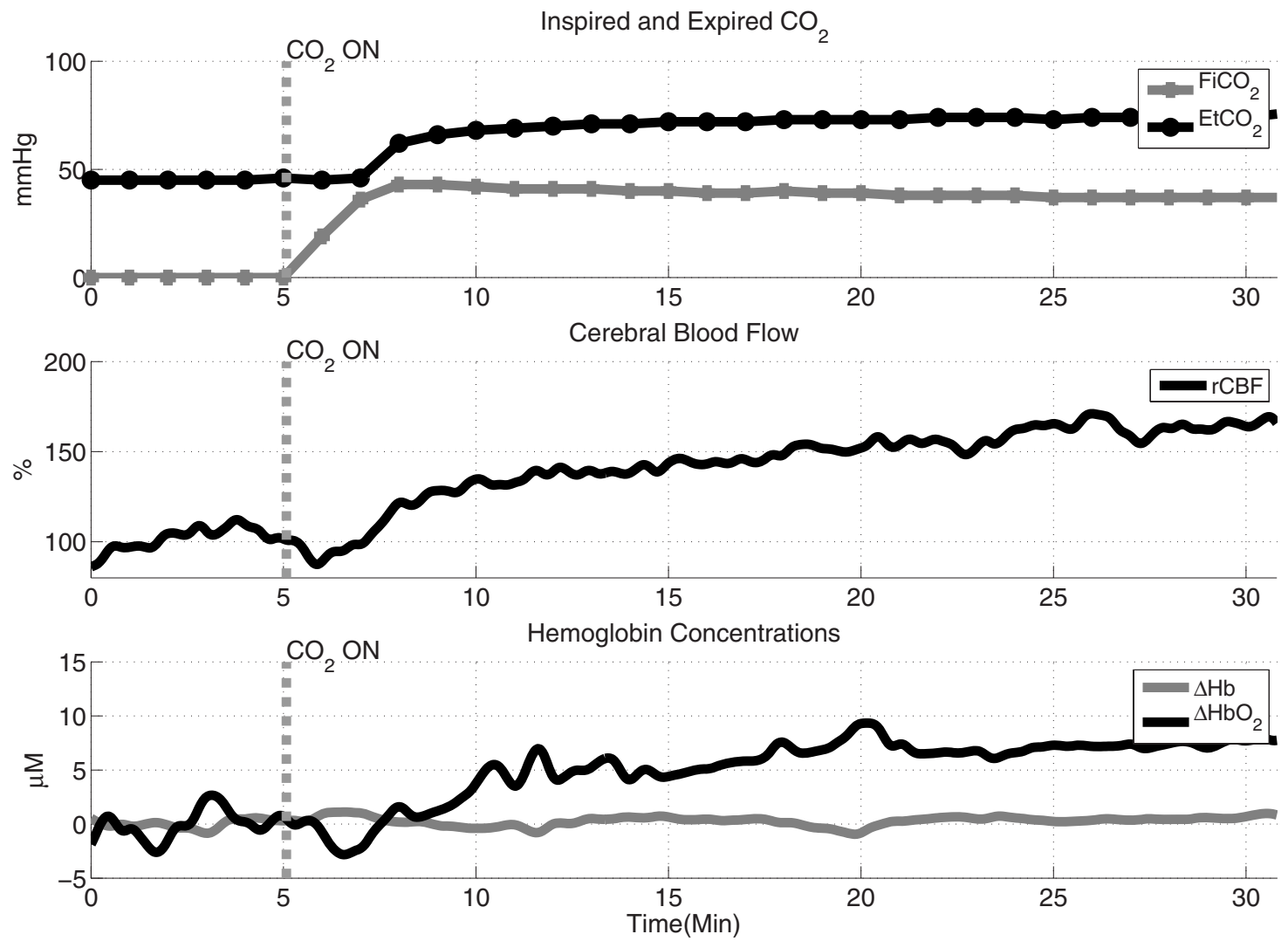

Fig. 3 Typical time traces of inspired and expired $\mathrm{CO}_{2}$ and cerebral hemodynamics during baseline and hypercapnia.

cal MRI interpretations were performed by a single pediatric neuroradiologist (RAZ) blinded to the patient's clinical information. Imaging parameters of the ASL-MRI scan were $\mathrm{FOV}=20 \mathrm{~cm}, 64 \times 64$ matrix, $\mathrm{TR} / \mathrm{TE}=3000 / 19 \mathrm{~ms}$, slice thickness $=5 \mathrm{~mm}$ and $1 \mathrm{~mm}$ gap. Eight slices were acquired using a gradient echo-planar imaging (EPI) sequence. A delay time $(1.2 \mathrm{~s})$ was applied between the saturation and excitation pulses to reduce transit artifacts. Because of large voxel sizes of ASL-MRI images of CBF, whole brain averages were used to compare against DCS.

\subsection{Overall Study Protocol}

MRI-compatible optical probes were designed with $\sim 12$-m-long optical fibers mounted on a flexible pad. The probe was placed on the neonate's forehead (Fig. 2). Concurrent baseline optical and baseline MRI perfusion measurements were obtained (Fig. 1). After completion of the baseline ASL-MRI measurements, supplemental $\mathrm{CO}_{2}$ was added to the fresh gas mixture to achieve an inspired $\mathrm{CO}_{2}\left(\mathrm{FiCO}_{2}\right)$ of $2.7 \%$ as measured by capnometry. Continuous optical data was acquired throughout the study. Structural brain MRI sequences were acquired for $\sim 10$ to $15 \mathrm{~min}$ after the initiation of supplemental $\mathrm{CO}_{2}$ and its equilibration. At the end of this period, a second set of ASL-MRI sequences were run to reflect the hypercarbic CBF. Blood gas samples were then drawn and analyzed to confirm a higher $\mathrm{PaCO}_{2}$. The hypercarbic gas mixture was discontinued after the completion of the hypercarbic CBF measurement, and the patient was transported back to the operating room directly for the surgery.

\subsection{Statistical Analysis}

Data from each subject was collected as a time series and normalized to a stable pre-hypercapnia period. In order to assess the hemodynamic changes during hypercapnia, the time period where end-tidal $\mathrm{CO}_{2}$ was stable was identified, and all optical data during that period were averaged. All data are reported as mean \pm SEM (standard error of the mean) when averaged over the population and as mean $\pm \sigma$ (standard error) when averaged over time for a single subject. Standard box-plots ${ }^{67}$ were used to visually explore the data.

Pearson's correlation coefficient $(R)$ and corresponding $p$-value (with $p<0.05$ considered as significant) were used to investigate correlations between modalities or parameters. Bland-Altman analysis ${ }^{68}$ was used to assess agreement between modalities (ASL-MRI and DCS measures of CBF) visually by identifying those measurements that lie outside the two standard-deviation range from the mean difference between results, and the slope is not significantly different from zero (with $p<0.05$ considered significant). Last, Lin's concordance correlation coefficient was used to investigate the accuracy of the agreement. ${ }^{69,70}$

A student's $t$-test was used to asses whether the estimated population mean of a hemodynamic change was significantly different from zero. $p<0.05$ was considered as the threshold for rejection of the null hypothesis.

\section{Results}

Figure 3 shows the time series of $\mathrm{FiCO}_{2}, \mathrm{EtCO}_{2}$, and the 
Distribution of The Optical \& MRI Data
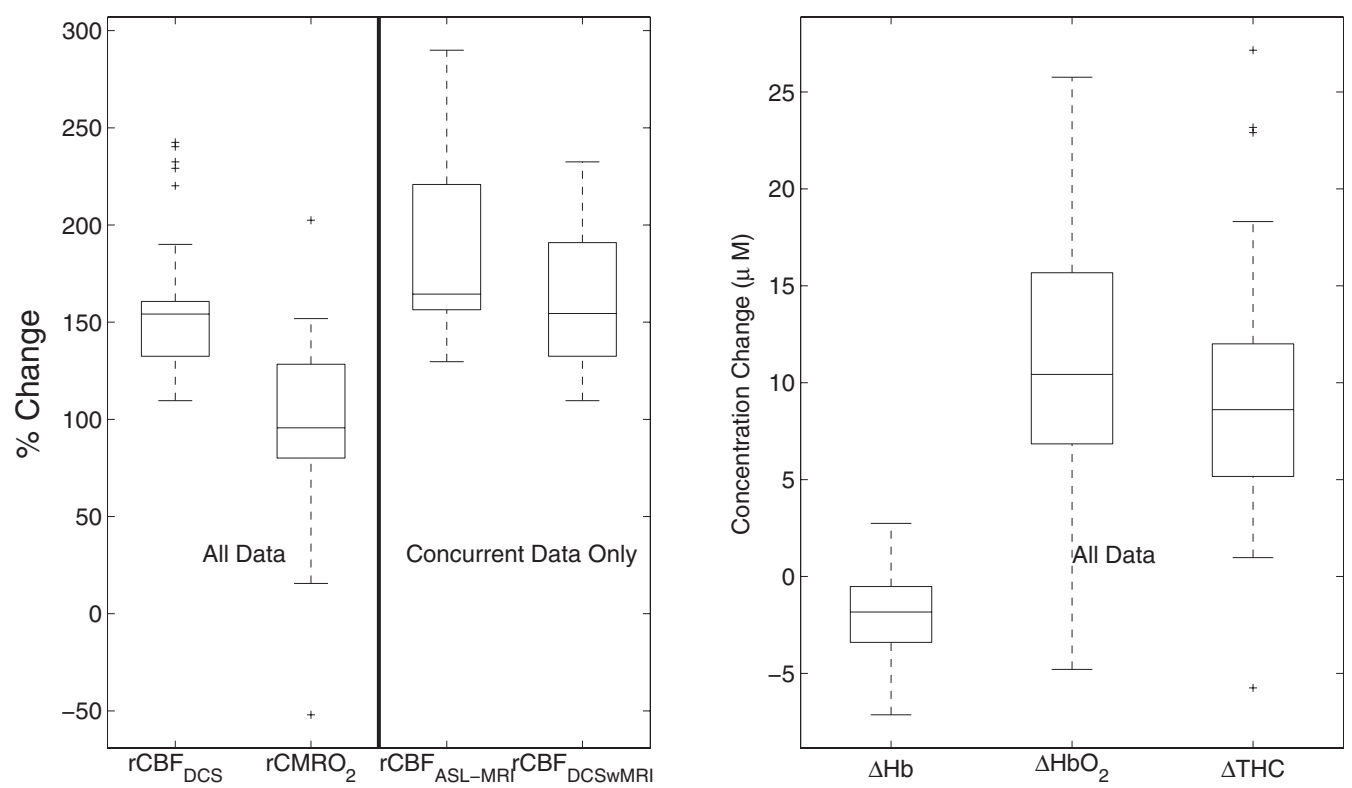

Fig. 4 Box-plots of $\mathrm{rCBF}_{A S L-M R I}, \mathrm{rCBF}_{D C S}, \mathrm{rCMRO}_{2}$ (\% change from baseline), $\Delta \mathrm{THC}, \Delta \mathrm{Hb}$, and $\Delta \mathrm{HbO}_{2}$ (concentration change from baseline) for whole data set $(n=33)$. Box-plot (top-left) shows the comparison of ASL and DCS $(n=12)$. Note that the lines in the boxes correspond to the lower quartile, median, and upper quartile. The mean corresponds to the center of the box. The dashed lines extend out to indicate the rest of the data within the interquartile distance. The crosses are the outliers.

optical data from a representative subject. Increased $\mathrm{InCO}_{2}$ led to increases in $\mathrm{EtCO}_{2}, \mathrm{rCBF}_{D C S}$ and $\triangle \mathrm{HbO}_{2}$. Blood pressure and arterial oxygen saturation remained relatively stable.

Figure 4 shows box-plots of population-averaged optical and MRI data. Significant increases were measured in $\mathrm{CBF}_{D C S}(158 \pm 6 \%, p<0.001, n=33), \mathrm{HbO}_{2}(11 \pm 1 \mu \mathrm{M}$, $p<0.001, n=33)$, and THC $(9 \pm 1 \mu \mathrm{M}, p<0.001, n=33)$. On the other hand, $\mathrm{Hb}$ decreased $(-2 \pm 0.4, \quad p<0.001$, $n=33)$, and $\mathrm{CMRO}_{2}$ was unaltered (98 $\left.\pm 8 \%, p=0.8, n=33\right)$. Concurrent measurements of $\mathrm{rCBF}_{A S L-M R I}(190 \pm 15 \%$, $p<0.001, n=12)$ and $\mathrm{rCBF}_{D C S}(164 \pm 12 \%, p<0.001$, $n=12$ ) demonstrated (Fig. 5) that $\mathrm{rCBF}_{D C S}$ and $\mathrm{rCBF}_{A S L-M R I}$ showed good correlation $(R=0.7, p=0.01, n=12)$ and good agreement (concordance correlation coefficient, $R_{c}=0.6$ ). Bland-Altman plots confirmed that all points lie within two

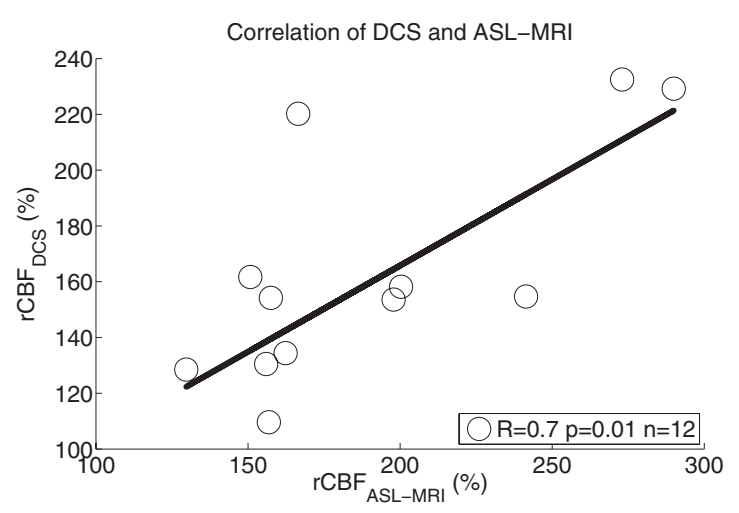

Fig. 5 Correlation between $\mathrm{rCBF}_{D C S}$ and $\mathrm{rCBF}_{A S L-M R I}$ is shown for each individual subject $(n=12)$. standard deviations from the mean difference between the results and that the slope is zero $(p=0.33)$.

We have also investigated whether NIRS measures of $\triangle T H C$ are related to $\mathrm{rCBF}_{D C S}$. As expected, a weaker $(R=0.5)$, but significant $(p=0.007)$ correlation between the two parameters was observed. The so-called Grubb exponent, ${ }^{71}$ which is the ratio of $\mathrm{THC}$ changes to $\mathrm{CBF}$ changes, was $0.39 \pm 0.04$, in reasonable agreement with the literature. ${ }^{38,71,72}$ We note that this value depends on the assumed baseline value for THC of $53 \mu \mathrm{M} .^{58-62}$

Vascular reactivity, defined as percent change in $\mathrm{CBF}$ per mmHg change in $\mathrm{PaCO}_{2}$, was measured to be $3.1 \pm 1.9 \%$ $\mathrm{CBF}$ change/mmHg CO $\mathrm{CO}_{2}$, well within the literature values of 1 to $9 \% \mathrm{CBF}$ change/mmHg $\mathrm{CO}_{2}$ in sick neonates. ${ }^{43,73-76}$ Vascular reactivity to $\mathrm{CO}_{2}$ was not found to depend on baseline $\mathrm{CBF}_{A S L-M R I}$ or baseline $\mathrm{PaCO}_{2}(p=0.1$ for both). The NIRS data was also in good agreement with previous NIRS studies on hypercapnia-induced changes in neonates. ${ }^{45,77,78}$

\section{Discussion}

This work demonstrates the feasibility of the DCS and NIRS hybrid method to measure blood flow, blood oxygenation, and total hemoglobin concentration in neonatal brains. NIRS is widely used, albeit mostly as a research tool ${ }^{11,12}$ - for example, a series of pioneering studies at our institution $^{45,61,77,79,80}$ and others ${ }^{46,81-84}$ showed that NIRS could play a role in monitoring cerebral oxygenation before, during, and after surgical interventions to patients with CHD. The application of DCS represents a new approach, adding noninvasive and direct measures of microvascular CBF to the arsenal of optical tools. Taken together, NIRS and DCS enable estimation of $\mathrm{rCMRO}_{2}$, thereby providing further insight into 
brain metabolism in CHD neonates. By studying a population of babies with complex congenital heart defects, we were able to utilize hypercapnia as a challenge to alter hemodynamics and validate DCS against ASL-MRI. If deployed during the presurgical period, these technologies should enable largescale studies of the relationship between cerebral autoregulation, $\mathrm{CO}_{2}$ reactivity, and other important physiological factors and neurological outcome.

ASL-MRI has recently been commercialized and validated against a large array of techniques. ${ }^{85}$ ASL-MRI is currently the only modality that can be utilized in neonates for microvascular CBF measurements. ASL-MRI provides full-brain images and can be applied repeatedly with minimal safety concerns. On the other hand, it is not a technology suitable for continuous monitoring, since it requires patient transport away from the safe confines of the intensive care unit.

Diffuse optical technologies in general, and DCS in particular, provide a promising alternative for continuous and bedside hemodynamic cerebral monitoring. Currently, DCS has been limited to measurements of relative CBF in cortex from few positions on the head. On the other hand, DCS accuracy does not depend on baseline $\mathrm{CBF}$, and the method does not require risky patient transport and appears capable of continuous monitoring for hours and even days. Therefore, DCS could provide complementary information to that available from ASL-MRI-e.g., DCS has the potential to study larger populations and healthy babies with minimal risk (e.g., without injection of a contrast agent, patient transfer, or anesthesia) in order to ferret out subtle differences between healthy and diseased response.

A somewhat surprising, although not entirely unexpected, result of this study is the demonstrated ability of these sick babies to respond to increased $\mathrm{CO}_{2}$. Our data agree with existing neonate data in the literature, ${ }^{43,73-76}$ but generally, these other studies measuring $\mathrm{CO}_{2}$ reactivity were performed on other sick neonates with various clinical conditions, since it is ethically difficult to justify the use of methods requiring contrast agents or anesthesia to measure local CBF and also the artificial induction of hypercapnia. Thus, we are unable to compare our data to the responses of healthy neonates. In the future, it should be possible to derive healthy neonate responses, since our optical methods are noninvasive and can be deployed at the bedside. We note that in comparison to healthy adults, ${ }^{86}$ our data show a wider range.

The NIRS data were also in agreement with the literature of hypercapnia-related changes in neonates with $\mathrm{CHD}^{45,77,78}$ and shows a well-behaved spread. $\triangle \mathrm{THC}$ was previously shown to be correlated to blood flow changes measured by Xenon-CT in neonates during hypercapnia. ${ }^{43}$ However, in comparison, we have observed only a weak correlation of $\triangle \mathrm{THC}$ with $\mathrm{rCBF}_{D C S}$.

Combined NIRS and DCS use is beneficial for two reasons: DCS analysis is improved by incorporating NIRS changes, and a more complete picture of cerebral well-being is derived by measuring $\mathrm{CBF}$ in combination with NIRSmeasured cerebral blood volume and oxygenation without the need for external tracers, without relying on assumptions that translate total hemoglobin concentration to cerebral blood volume, and without assumptions about how cerebral blood volume is then related to $\mathrm{CBF}$. The combined data can be used to measure changes in cerebral metabolic rate of oxygen; ${ }^{28,29,37,38}$ in fact, it has recently been demonstrated that NIRS and DCS combination provides a better estimate of changes in $\mathrm{CMRO}_{2}$ in premature infants than NIRS alone. ${ }^{35}$

Historically, it has often been assumed that $\mathrm{CMRO}_{2}$ does not change during hypercapnia. ${ }^{87}$ In fact, unchanging $\mathrm{CMRO}_{2}$ is assumed during hypercapnia and is used in functional MRI (fMRI) studies to calibrate the blood oxygen level dependent (BOLD) signals for measurement of $\mathrm{CMRO}_{2}$ during a functional task. ${ }^{88,89}$ However, several studies indicate increased or even reduced $\mathrm{CMRO}_{2}$ during hypercapnia in both healthy and disease states, leading to a continuing debate (see Ref. 90 and references therein). Very recently, for example, it was shown that spontaneous neuronal activity was reduced during hypercapnia, hinting at the possibility that $\mathrm{CMRO}_{2}$ may be reduced during hypercapnia. ${ }^{91}$ During surgical procedures with cardiopulmonary bypass where brain $\mathrm{CO}_{2}$ levels are managed according to different $\mathrm{pH}$ strategies, it was shown that different responses of $\mathrm{CMRO}_{2}$ to hypercapnia can be observed. ${ }^{92}$ During this artificially lowered baseline $\mathrm{CMRO}_{2}$ state, hypercapnia led to reductions in $\mathrm{CMRO}_{2}$ in one group but not in the other. In head injury patients, it was shown that $\mathrm{CMRO}_{2}$ was dependent on cerebral $\mathrm{CO}_{2}$ levels. ${ }^{93}$ Cerebral maturity is a known confounding factor, and studies have shown a direct correlation with cerebral $\mathrm{CO}_{2}$ and $\mathrm{CMRO}_{2}$ in immature animals. ${ }^{94}$ In immature rats, $\mathrm{CBF}$ was improved in response to hypercapnia during hypoxic-ischemic conditions. Presumably, the oxygen delivery was also improved under these conditions. ${ }^{95}$ This led to observed increases in glucose utilization and oxidative metabolism, and it was suggested that $\mathrm{CMRO}_{2}$ was lowered during the hypoxic-ischemic baseline as a neuroprotective action. In fact, it has been suggested that mild hypercapnia may be permissible for intensive care management of neonates in order to improve cerebral blood flow, oxygen delivery, oxygen consumption, and neurological outcome. ${ }^{96}$ Overall, the $\mathrm{CMRO}_{2}$ response to hypercapnia is very complex, and its measurement throughout the last decades relied on a multitude of modalities with their own strengths and weaknesses.

Our observations are in general agreement with the assumption of unchanged $\mathrm{CMRO}_{2}$. A weakness of our study was our reliance on literature data and other estimates for baseline values of $\mathrm{StO}_{2}$ and $\mathrm{THC}$, which have influenced our findings. To establish the effect of these assumptions on our estimates of $\mathrm{rCMRO}_{2}$, the baseline values were varied over a large range by assuming $S_{t} \mathrm{O}_{2 b l}$ to be correlated to baseline arterial concentration measured by co-oximetry for each neonate. We observed that small changes $(<10 \pm 4 \%)$ in $\mathrm{rCMRO}_{2}$ cannot be ruled out. Despite this weakness, the hybrid DCS and NIRS instrumentation is a relatively simpler and inexpensive device that could be readily deployed in hospital wards and clinics. From a diffuse optical technology development standpoint, further studies with an NIRS device capable of measuring absolute baseline values and potential studies to validate the assumptions that go into $\mathrm{rCMRO}_{2}$ calculations are now in place.

Last, we note that, to the best of our knowledge, no studies have been carried out on infants wherein anesthesia was varied and $\mathrm{CO}_{2}$ administration was repeated. Since it is expected that the relationship of hemodynamic response to $\mathrm{CO}_{2}$ and 
anesthesia is a mechanism that is species, age, and clinical condition dependent, it may be inaccurate to extrapolate from studies on animals. ${ }^{97,98}$ Therefore, in our conclusions, we rely on the fact that all infants were anesthetized in the same manner. Our work is accurate for comparison to other studies with this limitation.

\section{Conclusion}

We have recruited a cohort of neonates with complex congenital heart defects to study the cerebrovascular reactivity to increased $\mathrm{CO}_{2}$ (hypercapnia). By employing a hybrid diffuse correlation spectroscopy and near-infrared spectroscopy, we have measured changes during hypercapnia of oxyhemoglobin, deoxyhemoglobin, and total hemoglobin concentrations, cerebral blood flow ( $\mathrm{rCBF}$ ), and oxygen metabolism. In a subpopulation, we were able to obtain concurrent ASL-MRI data to validate the optical measurements of $\mathrm{rCBF}$.

We have shown that $\mathrm{rCBF}$ measurements by both modalities exhibit reasonable agreement. Hence, we have provided validation that diffuse correlation spectroscopy provides reliable measurements of changes in CBF in neonates. Furthermore, this population of neonates were shown to retain their cerebrovascular reactivity to hypercapnia. Combination of NIRS and DCS allowed us to study cerebral oxygen metabolism, which was unaltered in response to hypercapnia. Overall, the study demonstrates the potential to use hybrid diffuse optical probes on a critically ill neonatal population.

\section{Acknowledgments}

This study was supported by NIH Grant Nos. HL-57835, NS60653, NS-45839, RR-02305, EB-007610, HL-077699, HD26979, and NS-52380; Thrasher Research Fund (NR 0016); Fundació Cellex Barcelona; and June and Steve Wolfson Family Foundation. We acknowledge invaluable assistance from Dalton Hance and staff of the MRI facilities at Children's Hospital of Philadelphia.

\section{References}

1. Centers for Disease Control and Protection, "Improved national prevalence estimates for 18 selected major birth defects-United States, 1999-2001," Morbidity Mortality Weekly Report 54, 13011305 (2005).

2. S. P. Miller and P. S. McQuillen, "Neurology of congenital heart disease: insight from brain imaging," Arch. Dis. Child Fetal Neonatal Ed. 92, F435-437 (2007).

3. A. J. Marelli, A. S. Mackie, R. Ionescu-Ittu, E. Rahme, and L. Pilote, "Congenital heart disease in the general population: changing prevalence and age distribution," Circulation 115, 163-172 (2007).

4. M. T. Donofrio, Y. A. Bremer, R. M. Schieken, C. Gennings, L. D Morton, B. W. Eidem, F. Cetta, C. B. Falkensammer, J. C. Huhta, and C. S. Kleinman, "Autoregulation of cerebral blood flow in fetuses with congenital heart disease: the brain sparing effect," Pediatr. Cardiol. 24, 436-443 (2003).

5. D. J. Licht, J. Wang, D. W. Silvestre, S. C. Nicolson, L. M. Montenegro, G. Wernovsky, S. Tabbutt, S. M. Durning, D. M. Shera, J. W. Gaynor, T. L. Spray, R. R. Clancy, R. A. Zimmerman, and J. A. Detre, "Preoperative cerebral blood flow is diminished in neonates with severe congenital heart defects," J. Thorac. Cardiovasc. Surg. 128, 841-849 (2004).

6. O. B. Paulson, S. Strandgaard, and L. Edvinsson, "Cerebral autoregulation," Cerebrovasc Brain Metab. Rev. 2, 161-192 (1990).

7. R. B. Panerai, "Assessment of cerebral pressure autoregulation in humans-a review of measurement methods," Physiol. Meas 19. 305-338 (1998).

8. M. Wintermark, M. Sesay, E. Barbier, K. Borbely, W. P. Dillon, J. D.
Eastwood, T. C. Glenn, C. B. Grandin, S. Pedraza, J. F. Soustiel, T. Nariai, G. Zaharchuk, J. M. Caille, V. Dousset, and H. Yonas, "Comparative overview of brain perfusion imaging techniques," Stroke $\mathbf{3 6}$ 83-99 (2005).

9. A. Zauner, W. P. Daugherty, M. R. Bullock, and D. S. Warner, "Brain oxygenation and energy metabolism: part I-biological function and pathophysiology," Neurosurgery 51, 289-302 (2002).

10. A. Zauner and J. P. Muizelaar, "Measuring cerebral blood flow and metabolism," Chapter 11 in Head Injury, pp. 217-227, Chapman and Hall, London (1997).

11. A. Villringer and B. Chance, "Non-invasive optical spectroscopy and imaging of human brain function," Trends Neurosci. 20, 435-442 (1997).

12. E. M. C. Hillman, "Optical brain imaging in vivo: techniques and applications from animal to man," J. Biomed. Opt. 12051402 (2007).

13. J. E. Brazy, D. V. Lewis, M. H. Mitnick, and F. F. Jobsis, "Noninvasive monitoring of cerebral oxygenation in preterm infants: preliminary observations," Pediatrics 75, 217-225 (1985).

14. J. E. Brazy and D. V. Lewis, "Changes in cerebral blood volume and cytochrome AA3 during hypertensive peaks in preterm infants," $J$. Pediatr. 108, 983-987 (1986).

15. H. U. Bucher, A. D. Edwards, A. E. Lipp, and G. Duc, "Comparison between near infrared spectroscopy and 133Xenon clearance for estimation of cerebral blood flow in critically ill preterm infants," $P e$ diatr. Res. 33, 56-59 (1993).

16. M. Cope, The Development of a Near-Infrared Spectroscopy System and Its Application for Noninvasive Monitoring of Cerebral Blood and Tissue Oxygenation in the Newborn Infant, University College London, London (1991).

17. M. Cope and D. T. Delpy, "System for long-term measurement of cerebral blood flow and tissue oxygenation on newborn infants by infra-red transillumination," Med. Biol. Eng. Comput. 26, 289-294 (1988).

18. D. T. Delpy, M. C. Cope, E. B. Cady, J. S. Wyatt, P. A. Hamilton, P. L. Hope, S. Wray, and E. O. Reynolds, "Cerebral monitoring in newborn infants by magnetic resonance and near infrared spectroscopy," Scand. J. Clin. Lab Invest Suppl. 188, 9-17 (1987).

19. S. R. Hintz, D. A. Benaron, A. M. Siegel, D. K. Stevenson, and D. A. Boas, "Bedside functional imaging of the premature infant brain during passive motor activation," J. Perinat. Med. 29(4), 335-343 (2001).

20. J. C. Hebden, "Advances in optical imaging of the newborn infant brain," Psychophysiology 40, 501-510 (2003).

21. A. D. Edwards, C. Richardson, M. Cope, J. S. Wyatt, D. T. Delpy, and E. O. R. Reynolds, "Cotside measurement of cerebral blood flow in ill newborn infants by near infrared spectroscopy," Lancet 332 770-771 (1988).

22. W. M. Kuebler, "How NIR is the future in blood flow monitoring," $J$. Appl. Physiol. 104, 905-906 (2008).

23. A. J. Wolfberg and A. J. du Plessis, "Near-infrared spectroscopy in the fetus and neonate," Clin. Perinatol. 33, 707-728 (2006).

24. D. A. Boas, L. E. Campbell, and A. G. Yodh, "Scattering and imaging with diffusing temporal field correlations," Phys. Rev. Lett. $\mathbf{7 5}$ 1855-1858 (1995).

25. D. A. Boas and A. G. Yodh, "Spatially varying dynamical properties of turbid media probed with diffusing temporal light correlation," $J$. Opt. Soc. Am. A 14, 192-215 (1997).

26. D. J. Pine, D. A. Weitz, P. M. Chaikin, and E. Herbolzheimer, "Diffusing-wave spectroscopy," Phys. Rev. Lett. 60, 1134-1137 (1988).

27. G. Maret and P. E. Wolf, "Multiple light scattering from disordered media. The effect of Brownian motion of scatterers," Z. Phys. B 65, 409-413 (1987).

28. T. Durduran, "Non-invasive measurements of tissue hemodynamics with hybrid diffuse optical methods," Ph.D. Dissertation, Univ. of Pennsylvania (2004).

29. T. Durduran, G. Yu, M. G. Burnett, J. A. Detre, J. H. Greenberg, J. Wang, C. Zhou, and A. G. Yodh, "Diffuse optical measurements of blood flow, blood oxygenation, and metabolism in human brain during sensorimotor cortex activation," Opt. Lett. 29, 1766-1768 (2004)

30. J. Li, G. Dietsche, D. Iftime, S. E. Skipetrov, G. Maret, T. Elbert, B. Rockstroh, and T. Gisler, "Noninvasive detection of functional brain activity with near-infrared diffusing-wave spectroscopy," J. Biomed. Opt. 10, 044002 (2005).

31. G. Yu, T. Durduran, C. Zhou, H. W. Wang, M. E. Putt, M. Saunders, 
C. M. Seghal, E. Glatstein, A. G. Yodh, and T. M. Busch, "Noninvasive monitoring of murine tumor blood flow during and after photodynamic therapy provides early assessment of therapeutic efficacy," Clin. Cancer Res. 11, 3543-3552 (2005).

32. G. Yu, T. Floyd, T. Durduran, C. Zhou, J. J. Wang, J. A. Detre, and A. G. Yodh, "Validation of diffuse correlation spectroscopy for muscle blood flow with concurrent arterial-spin-labeling perfusion," Opt. Express 15, 1064-1075 (2007).

33. E. M. Buckley, N. M. Cook, T. Durduran, M. N. Kim, C. Zhou, R Choe, G. Yu, S. Shultz, C. M. Sehgal, D. J. Licht, P. H. Arger, M. E. Putt, H. H. Hurt, and A. G. Yodh, "Cerebral hemodynamics in preterm infants during positional intervention measured with diffuse correlation spectroscopy and transcranial doppler ultrasound," Opt. Express 17, 12571-12581 (2009).

34. C. Zhou, S. Eucker, T. Durduran, G. Yu, J. Ralston, S. H. Friess, R N. Ichord, S. S. Margulies, and A. G. Yodh, "Diffuse optical monitoring of hemodynamic changes in piglet brain with closed head injury," J. Biomed. Opt. 14, 034015 (2009).

35. N. Roche-Labarbe, S. A. Carp, A. Surova, M. Patel, D. A. Boas, P. E. Grant, and M. Franceschini, "Noninvasive optical measures of CBV, $\mathrm{StO} 2, \mathrm{CBF}$ index, and $\mathrm{rCMRO} 2$ in human premature neonates' brains in the first six weeks of life (p NA)," Hum. Brain Mapp 31(3), 341352 (2009)

36. M. N. Kim, T. Durduran, S. Frangos, B. L. Edlow, E. M. Buckley, E. M. Heather, C. Zhou, G. Yu, R. Choe, M. E, R. L. Wolf, J. H. Woo, M. S. Grady, J. H. Greenberg, J. M. Levine, A. G. Yodh, J. A. Detre, and W. A. Kofke, "Noninvasive measurement of cerebral blood flow and blood oxygenation using near-infrared and diffuse correlation spectroscopies in critically brain-injured adults," Neurocritical Care 12(2), 173-180 (2009)

37. J. P. Culver, T. Durduran, D. Furuya, C. Cheung, J. H. Greenberg, and A. G. Yodh, "Diffuse optical tomography of cerebral blood flow, oxygenation, and metabolism in rat during focal ischemia," J. Cereb. Blood Flow Metab. 23, 911-924 (2003).

38. C. Cheung, J. P. Culver, K. Takahashi, J. H. Greenberg, and A. G. Yodh, "In vivo cerebrovascular measurement combining diffuse nearinfrared absorption and correlation spectroscopies," Phys. Med. Biol. 46, 2053-2065 (2001).

39. C. Zhou, G. Yu, D. Furuya, J. H. Greenberg, A. G. Yodh, and T. Durduran, "Diffuse optical correlation tomography of cerebral blood flow during cortical spreading depression in rat brain," Opt. Express 14, 1125-1144 (2006).

40. T. Durduran, C. Zhou, B. L. Edlow, G. Yu, R. Choe, M. N. Kim, B. L. Cucchiara, M. E. Putt, Q. Shah, S. E. Kasner, J. H. Greenberg, A. G. Yodh, and J. A. Detre, "Transcranial optical monitoring of cerebrovascular hemodynamics in acute stroke patients," Opt. Express 17, 3884-3902 (2009).

41. S. S. Kety and C. F. Schmidt, "The nitrous oxide method for the quantitative determination of cerebral blood flow in man: theory, procedure, and normal values," J. Clin. Invest. 27, 476-483 (1948).

42. S. Ashwal, W. Stringer, L. Tomasi, S. Schneider, J. Thompson, and R. Perkin, "Cerebral blood flow and carbon dioxide reactivity in children with bacterial meningitis," J. Pediatr. 117, 523-530 (1990).

43. O. Pryds, G. Greisen, L. L. Skov, and B. Friis-Hansen, "Carbon dioxide-related changes in cerebral blood volume and cerebral blood flow in mechanically ventilated preterm neonates: comparison of near-infrared spectrophotometry and 133Xenon clearance," Pediatr. Res. 27, 445-449 (1990).

44. S. Ashwal, R. M. Perkin, J. R. Thompson, L. G. Tomasi, D. van Stralen, and S. Schneider, "CBF and $\mathrm{CBF} / \mathrm{PCO} 2$ reactivity in childhood strangulation," Pediatr. Neurol. 7, 369-374 (1991).

45. S. Tabbutt, C. Ramamoorthy, L. M. Montenegro, S. M. Durning, C. D. Kurth, J. M. Steven, R. I. Godinez, T. L. Spray, G. Wernovsky, and S. C. Nicolson, "Impact of inspired gas mixtures on preoperative infants with hypoplastic left heart syndrome during controlled ventilation," Circulation 104, I-159-164 (2001).

46. F. Kern, R. Ungerleider, T. Quill, B. Baldwin, W. White, J. Reves, and W. Greeley, "Cerebral blood flow response to changes in arterial carbon dioxide tension during hypothermic cardiopulmonary bypass in children," J. Thorac. Cardiovasc. Surg. 101, 618-622 (1991).

47. J. A. Stockwell, R. F. Goldstein, R. M. Ungerleider, F. H. Kern, J. N. Meliones, and W. J. Greeley, "Cerebral blood flow and carbon dioxide reactivity in neonates during venoarterial extracorporeal life support," Crit. Care Med. 24, 155-162 (1996).

48. M. J. Miranda, K. Olofsson, and K. Sidaros, "Noninvasive measure- ments of regional cerebral perfusion in preterm and term neonates by magnetic resonance arterial spin labeling," Pediatr. Res. 60, 359-363 (2006).

49. C. J. Petit, J. J. Rome, G. Wernovsky, S. E. Mason, D. M. Shera, S. C. Nicolson, L. M. Montenegro, S. Tabbutt, R. A. Zimmerman, and D. J. Licht, "Preoperative brain injury in transposition of the great arteries is associated with oxygenation and time to surgery, not balloon atrial septostomy," Circulation 119, 709-716 (2009).

50. J. Li, G. Zhang, H. Holtby, A. Guerguerian, S. Cai, T. Humpl, C. A. Caldarone, A. N. Redington, and G. S. Van Arsdell, "The influence of systemic hemodynamics and oxygen transport on cerebral oxygen saturation in neonates after the Norwood procedure," J. Thorac. Cardiovasc. Surg. 135, 83-90.e2 (2008).

51. D. J. Licht, D. M. Shera, R. R. Clancy, G. Wernovsky, L. M. Montenegro, S. C. Nicolson, R. A. Zimmerman, T. L. Spray, J. W. Gaynor, and A. Vossough, "Brain maturation is delayed in infants with complex congenital heart defects," J. Thorac. Cardiovasc. Surg. 137, 529-537 (2009)

52. P. van der Zee, M. Cope, S. R. Arridge, M. Essenpreis, L. A. Potter, A. D. Edwards, J. S. Wyatt, D. C. McCormick, S. C. Toth, E. O. R. Reynolds, and D. T. Delpy, "Experimentally measured optical pathlengths for the adult's head, calf, and forearm and the head of the newborn infant as a function of interoptode spacing," Adv. Exp. Med. Biol. 316, 143-153 (1992).

53. A. Duncan, J. H. Meek, M. Clemence, C. E. Elwell, L. Tyszczuk, M. Cope, and D. T. Delpy, "Optical pathlength measurements on adult head, calf, and forearm and the head of the newborn infant using phase-resolved optical spectroscopy," Phys. Med. Biol. 40, 295-304 (1995).

54. M. Kohl, C. Nolte, H. R. Heekeren, S. Horst, U. Scholz, H. Obrig, and A. Villringer, "Determination of the wavelength dependence of the differential pathlength factor from near-infrared pulse signals," Phys. Med. Biol. 43, 1771-1782 (1998).

55. R. B. Buxton and L. R. Frank, "A model for the coupling between cerebral blood flow and oxygen metabolism during neural stimulation," J. Cereb. Blood Flow Metab. 17, 64-72 (1997)

56. K. J. Friston, A. Mechelli, R. Turner, and C. J. Price, "Nonlinear responses in fMRI: the balloon model, Volterra kernels, and other hemodynamics," Neuroimage 12, 466-477 (2000).

57. A. Gjedde, "The relation between brain function and cerebral blood flow and metabolism," in Cerebrovascular Disease, H. Hunt Batjer L. R. Caplan, L. Friberg, R. G. Greenlee, Jr., T. A. Kropitnik, Jr., and W. L. Young, Eds., pp. 23-40, Lippincott-Raven, Philadelphia (1997).

58. S. Ijichi, T. Kusaka, K. Isobe, K. Okubo, K. Kawada, M. Namba, H. Okada, T. Nishida, T. Imai, and S. Itoh, "Developmental changes of optical properties in neonates determined by near-infrared timeresolved spectroscopy," Pediatr. Res. 58, 568-573 (2005).

59. M. A. Franceschini, S. Thaker, G. Themelis, K. K. Krishnamoorthy, H. Bortfeld, S. G. Diamond, D. A. Boas, K. Arvin, and P. E. Grant, "Assessment of infant brain development with frequency-domain near-infrared spectroscopy," Pediatr. Res. 61, 546-551 (2007).

60. J. Zhao, H. S. Ding, X. L. Hou, C. L. Zhou, and B. Chance, "In vivo determination of the optical properties of infant brain using frequency-domain near-infrared spectroscopy," J. Biomed. Opt. 10 , 024028 (2005)

61. C. D. Kurth, J. L. Steven, L. M. Montenegro, H. M. Watzman, J. W Gaynor, T. L. Spray, and S. C. Nicolson, "Cerebral oxygen saturation before congenital heart surgery," Ann. Thorac. Surg. 72, 187-192 (2001).

62. K. N. Fenton, K. Freeman, K. Glogowski, S. Fogg, and K. F. Duncan, "The significance of baseline cerebral oxygen saturation in children undergoing congenital heart surgery," Am. J. Surg. 190, 260-263 (2005).

63. H. M. Watzman, C. D. Kurth, L. M. Montenegro, J. Rome, J. M. Steven, and S. C. Nicolson, "Arterial and venous contributions to near-infrared cerebral oximetry," Anesthesiology 93, 947-953 (2000).

64. J. Wang and D. J. Licht, "Pediatric perfusion MR imaging using arterial spin labeling," Neuroimaging Clin. N. Am. 16, 149-167 (2006).

65. J. Wang, D. J. Licht, D. W. Silvestre, and J. A. Detre, "Why perfusion in neonates with congenital heart defects is negative-technical issues related to pulsed arterial spin labeling," Magn. Reson. Imaging 24, 249-254 (2006).

66. J. Wang, D. J. Licht, G.-H. Jahng, C.-S. Liu, J. T. Rubin, J. Hasel- 
grove, R. A. Zimmerman, and J. A. Detre, "Pediatric perfusion imaging using pulsed arterial spin labeling," J. Magn. Reson Imaging 18, 404-413 (2003).

67. J. W. Tukey, Exploratory Data Analysis, Addison-Wesley, Reading, MA (1977).

68. J. M. Bland and D. G. Altman, "Comparing methods of measurement: why plotting difference against standard method is misleading," Lancet 346, 1085-1087 (1995).

69. L. I. Lin, "A concordance correlation coefficient to evaluate reproducibility," Biometrics 45, 255_268 (1989).

70. L. I. K. Lin, "A note on the concordance correlation coefficient," Biometrics 56, 324-325 (2000).

71. R. L. J. Grubb, M. Raichle, J. O. Eichling, and M. M. Ter-Pogossian, "The effects of changes in $\mathrm{PaCO}_{2}$ on cerebral blood volume, blood flow, and vascular mean transit time," Stroke 5, 630-639 (1974).

72. T. S. Leung, M. M. Tachtsidis, I. Tisdall, C. Pritchard, M. Smith, and C. E. Elwell, "Estimating a modified Grubb's exponent in healthy human brains with near-infrared spectroscopy and transcranial Doppler," Physiol. Meas 30, 1-12 (2009).

73. O. Pryds, G. Greisen, H. Lou, and B. Friis-Hansen, "Heterogeneity of cerebral vasoreactivity in preterm infants supported by mechanical ventilation," J. Pediatr. 115, 638-645 (1989).

74. M. I. Levene, D. Shortland, N. Gibson, and D. H. Evans, "Carbon dioxide reactivity of the cerebral circulation in extremely premature infants: effects of postnatal age and indomethacin," Pediatr. Res. 24, 175-179 (1988).

75. G. Greisen and W. Trojaborg, "Cerebral blood flow, $\mathrm{PaCO}_{2}$ changes, and visual evoked potentials in mechanically ventilated preterm infants," Acta Paediatr. Scand. 76, 394-400 (1987).

76. O. Baenziger, M. Moenkhoff, C. G. Morales, K. Waldvogel, M. Wolf, H. Bucher, and S. Fanconi, "Impaired chemical coupling of cerebral blood flow is compatible with intact neurological outcome in neonates with perinatal risk factors," Biol. Neonate 75, 9-17 (1999).

77. C. Ramamoorthy, S. Tabbutt, C. D. Kurth, J. M. Steven, L. M. Montenegro, S. Durning, G. Wernovsky, J. W. Gaynor, T. L. Spray, and S. C. Nicolson, "Effects of inspired hypoxic and hypercapnic gas mixtures on cerebral oxygen saturation in neonates with univentricular heart defects," Anesthesiology 96, 283-288 (2002).

78. J. S. Wyatt, A. D. Edwards, M. Cope, D. T. Delpy, D. C. McCormick, A. Potter, and E. O. Reynolds, "Response of cerebral blood volume to changes in arterial carbon dioxide tension in preterm and term infants," Pediatr. Res. 29, 553-557 (1991).

79. T. Durduran, C. Zhou, M. N. Kim, E. M. Buckley, G. Yu, R. Choe, S. M. Durning, S. Mason, L. M. Montenegro, S. C. Nicholson, R. A Zimmerman, J. J. Wang, J. A. Detre, A. G. Yodh, and D. J. Licht, "Validation of diffuse correlation spectroscopy for non-invasive, continuous monitoring of $\mathrm{CBF}$ in neonates with congenital heart defects," in Annu. Meeting American Neurological Association, Abstract 299, American Neurological Association, Salt Lake City, UT (2008).

80. C. D. Kurth, J. Steven, S. Nicolson, and M. Jacobs, "Cerebral oxygenation during cardiopulmonary bypass in children," J. Thorac. Cardiovasc. Surg. 113, 71-79 (1997).

81. P. Fallon, I. Roberts, F. J. Kirkham, M. J. Elliott, A. Lloyd-Thomas, R. Maynard, and A. D. Edwards, "Cerebral hemodynamics during cardiopulmonary bypass in children using near-infrared spectroscopy," Ann. Thorac. Surg. 56, 1473-1477 (1993).

82. T. Takami, H. Yamamura, K. Inai, Y. Nishikawa, Y. Takei, A. Hoshika, and M. Nakazawa,"Monitoring of cerebral oxygenation during hypoxic gas management in congenital heart disease with incrased pulmonary blood flow," Pediatr. Res. 58, 521-524 (2005).

83. P. S. McQuillen, A. J. Barkovich, S. E. G. Hamrick, M. Perez, P. Ward, D. V. Glidden, A. Azakie, T. Karl, and S. P. Miller, "Temporal and anatomic risk profile of brain injury with neonatal repair of congenital heart defects," Stroke 38, 736-741 (2007).

84. I. Roberts, P. Fallon, F. Kirkham, P. Kirshbom, C. Cooper, M. Elliott, and A. Edwards, "Measurement of cerebral blood flow during cardiopulmonary bypass with near-infrared spectroscopy," J. Thorac. Cardiovasc. Surg. 115, 94-98 (1998).

85. E. T. Petersen, I. Zimine, Y.-C. L. Ho, and X. Golay, "Non-invasive measurement of perfusion: a critical review of arterial spin labelling techniques," Br. J. Radiol. 79, 688-701 (2006).

86. L. Sokoloff, "The effects of carbon dioxide on the cerebral circulation," Anesthesiology 21, 664-673 (1960).

87. B. K. Siesjo, "Carbon dioxide in brain energy metabolism," in Brain Energy Metabolism, pp. 131-150, Wiley, New York (1978).

88. R. D. Hoge, J. Atkinson, B. Gill, G. R. Crelier, S. Marrett, and G. B. Pike, "Investigation of BOLD signal dependence on cerebral blood flow and oxygen consumption: the deoxyhemoglobin dilution model," Magn. Reson. Med. 42, 849-863 (1999).

89. T. L. Davis, K. K. Kwong, R. M. Weisskoff, and B. R. Rosen, "Calibrated functional MRI: mapping the dynamics of oxidative metabolism," Proc. Natl. Acad. Sci. U.S.A. 95, 1834-1839 (1998).

90. B. Siesjo, "Cerebral metabolic rate in hypercarbia-A controversy; editorial view," Anesthesiology 52, 461-465 (1980).

91. A. C. Zappe, K. Uludag, A. Oeltermann, K. Ugurbil, and N. K. Logothetis, "The influence of moderate hypercapnia on neural activity in the anesthetized nonhuman primate," Cereb. Cortex 18, 2666-2673 (2008).

92. D. S. Prough, A. T. Rogers, D. M. S. Stump, G. P. Gravlee, and C. Taylor, "Hypercarbia depresses cerebral oxygen consumption during cardiopulmonary bypass," Stroke 21, 1162-1166 (1990).

93. W. D. Obrist, G. L. Clifton, C. S. Robertson, and T. W. Langfitt, "Cerebral metabolic changes induced by hyperventilation in acute head injury," in Cerebral Vascular Disease, J. S. Meyer, K. Lechaer, M. Reivich, and E. O. Ott, Eds., pp. 251-255, Elsevier Science Publishers, New York (1987).

94. J. H. Reuter and T. A. Disney, "Regional cerebral blood flow and cerebral metabolic rate of oxygen during hyperventilation in the newborn dog," Pediatr. Res. 20, 1102-1106 (1986).

95. R. C. Vannucci, R. M. Brucklacher, and S. J. Vannucci, "Effect of carbon dioxide on cerebral metabolism during hypoxia-ischemia in the immature rat," Pediatr. Res. 42, 24-29 (1997).

96. R. P. Jankov and A. K. Tanswell, "Hypercapnia and the neonate," Acta Paediatr. 97, 1502-1509 (2008).

97. A. Dahan and L. Teppema, "Influence of low-dose anaesthetic agents on ventilatory control: where do we stand?," Br. J. Anaesth. 83, 204209 (1999).

98. B. K. Siesjo, Brain Energy Metabolism, John Wiley \& Sons Ltd (1978). 